\title{
Análise dinâmica da volatilidade dos retornos do Ibovespa: uma aplicação do modelo autorregressivo de mudanças markovianas
}

O presente artigo utiliza o modelo Markov Switching Autorregressivo de dois estados desenvolvido por Hamilton (1989), para capturar mudanças de regime tanto na média quanto na variância dos retornos do lbovespa, entre janeiro de 2000 e maio de 2020 . A evidência empírica indica probabilidades de transição sugerindo que apenas eventos podem mudar a série de um regime de baixa volatilidade para um regime de alta volatilidade e vice-versa. Verificou-se que os resultados do modelo MS(2)-AR(1) detectaram momento das mudanças de regimes dos retornos, por causa da eleição presidencial, crises financeiras 2008 e a pandemia (coronavírus 2020).

Palavras-chave: Ibovespa; Modelo Markov Switching Autorregressivo; Probabilidade de Transição; Duração de Regime.

\section{Dynamic analysis of the volatility of Ibovespa returns: an application of the Markov Switching autoregressive model}

The present article uses the two-state Markov Switching autoregressive model developed by Hamilton (1989), to capture regime changes both in the mean and in the variance of Ibovespa returns, between January 2000 and May 2020. The empirical evidence indicates probabilities of transition suggesting that only events can change the series from a low volatility regime to a high volatility regime and contrary. It was found that the results of the MS(2)-AR(1) model detected moment of changes in returns regimes, because of the presidential election, financial crises 2008 and the pandemic (coronavírus 2020).

Keywords: Ibovespa; Markov Switching Autoregressive Model; Probability of Transition; Regime Duration.

Topic: Finanças Empresariais

Reviewed anonymously in the process of blind peer.

Carlos Alberto Gonçalves da Silva

Centro Federal de Educação Tecnológica Celso Suckow da Fonseca, Brasil http://lattes.cnpq.br/4301394305558781

http://orcid.org/0000-0002-6827-5073

ca7gon@gmail.com
Received: 07/10/2020

Approved: 21/12/2020
Referencing this:

SILVA, C. A. G.. Análise dinâmica da volatilidade dos retornos do Ibovespa: uma aplicação do modelo autorregressivo de mudanças markovianas. Revista Brasileira de Administração Científica, v.11, n.4, p.1-9, 2020. DOI: http://doi.org/10.6008/CBPC2179684X.2020.004.0001 


\section{INTRODUÇÃO}

O presente artigo utilizou-se modelo Markov Switching Autorregressivo de dois estados desenvolvido por Hamilton (1989), para capturar mudanças de regime tanto na média quanto na variância dos retornos do Ibovespa compreendendo o período entre janeiro de 2000 e maio de 2020.

A seguir realiza-se um breve comentário de alguns trabalhos aplicando modelos de Markov Switching conjuntamente com modelos ARIMA, VAR e GARCH. Schwert (1989) considera em que as rentabilidades do mercado acionário podem ter uma variação de alta e baixa determinadas por um processo de Markov de dois estados.

Hamilton (1989) utilizam um modelo com mudanças, no que diz respeito à volatilidade. Segundo os autores o modelo com mudança de regime, aplicado aos retornos do mercado acionário americano, ajustase melhor aos dados do que os modelos ARCH sem mudança de regime.

Krolzig (1997) com base no estudo realizado por Hamilton (1989) acrescentou modelos multivariados (VAR). O estudo analisou os ciclos econômicos internacionais com atenção especial ao caso da Alemanha. Foram encontradas relações de causalidade entre as fases de ciclos econômicos entre diferentes economias.

Ang et al. (2002) aplicaram através de modelo não linear nas taxas de juros dos EUA, Alemanha e Reino Unido. Assim, os autores mostraram que os regimes das taxas de juros correspondem razoavelmente bem com os ciclos econômicos dos EUA, tendo extrema importância para estudar os efeitos dos choques da política monetária sobre a economia.

Ismail et al. (2006) usaram teste de mudança de regime em seu estudo para detectar características não lineares nas taxas de câmbio de três países Asiáticos . Eles detectaram que a hipótese nula de linearidade é rejeitada e há evidências de quebras estruturais nas séries das taxas de câmbio.

Wink Júnior et al. (2014) testaram a hipótese de não linearidade da sensibilidade do retorno de ativos de empresas gaúchas em diferentes regimes markovianos de risco: períodos de crise e de estabilidade. Consideraram três ativos de empresas gaúchas negociáveis na Bolsa de Valores de São Paulo (Bovespa). Os resultados mostraram que o modelo não linear (MS-CAPM) é o mais adequado. Além disso, as evidências de que os ativos são mais suscetíveis às variações macroeconômicas em tempos de crise do que em períodos de estabilidade.

Mahjoub et al. (2019) aplicaram o modelo Markov Switching com dois regimes, para identificar os períodos de formação e explosão de bolhas especulativas no mercado de capitais do Irâ. O regime 1 é o crescimento de bolhas e o estágio de explosão e o regime 2 identifica a perda de bolhas. O resultado da pesquisa mostra que índice de ações do mercado de capitais iraniano no período analisado teve 18 meses de bolhas e 66 meses de equilíbrio.

Vários autores desenvolveram trabalhos utilizando-se modelos Markov Switching, pode-se citar Kim (1994), Norden et al. (1995), Oliveira et al. (2018), Krolzig et al. (2004), Assoe (1998) e Safaei et al. (2012). 


\section{REVISÃO TEÓRICA}

\section{O modelo Markov Switching autorregressivo}

O modelo Markov Switching (MS) foi introduzido por Hamilton (1989). O modelo Markov Switching autorregressivo (MS-AR) de dois estados (ou regimes) com um processo de ordem AR (p) é escrito como:

$$
y_{t}=\left\{\begin{array}{cc}
c_{1}+\alpha_{11} \gamma_{t-1}+\ldots \ldots \ldots \ldots \ldots+\alpha_{p 1} \gamma_{t-p}+\varepsilon_{t} & S_{t}=1 \\
c_{2}+\alpha_{12} \gamma_{t-1}+\ldots \ldots \ldots \ldots \ldots .+\alpha_{p 2} \gamma_{t-p}+\varepsilon_{t} & S_{t}=2
\end{array}\right\}
$$

Onde os regimes do modelo (1) são indexados por $S_{t}$. Os parâmetros da parte autorregressivas e do intercepto dependem do regime no tempo $t$. Os regimes são assumidos como variáveis não observáveis discretas. 0 regime 1 descreve os períodos de tendência de baixa volatilidade do lbovespa e o regime 2 indica os períodos de tendência de alta volatilidade do Ibovespa.

A transição de probabilidades entre os regimes é realizada por um processo de Markov de primeira ordem da seguinte maneira:

$$
\left.\rho_{i j}=\operatorname{Pr}\left(S_{t}=j\right) \mid S_{t-1}=i\right)
$$

em que $\rho_{i j}$ refere-se à probabilidade de estar no regime $j$ dado que o processo encontra-se no regime $i$, onde

$$
\sum_{i=1}^{N} \rho_{i j}=1 \text { para todo } i, j \in(1,2, \ldots . . . N) .
$$

As probabilidades de transição em uma matriz quadrada de ordem $N$, conhecida como matriz de transição e denotada por $P$, tem a seguinte forma:

$$
P=\left[\begin{array}{ll}
\rho_{11} & \rho_{12} \\
\rho_{21} & \rho_{22}
\end{array}\right]
$$

$$
\begin{aligned}
& \text { Onde } \\
& \rho_{11}=P\left[s_{t}=1 ; s_{t+1}=1\right] \\
& \rho_{12}=P\left[s_{t}=2 ; s_{t+1}=1\right] \\
& \rho_{21}=P\left[s_{t}=1 ; s_{t+1}=2\right] \\
& \rho_{22}=P\left[s=2 ; s_{t+1}=2\right] \\
& \rho_{11}+\rho_{12}=1 \quad \text { e } \quad \rho_{21}+\rho_{22}=1
\end{aligned}
$$

Assim, admite-se que a matriz de transição é irredutível e ergódica (se um dos valores próprios da matriz de transição for igual à unidade e todos os outros autovalores estiverem dentro do círculo unitário). Com essas condições, existe uma distribuição de probabilidade estacionária dos regimes (KROLZIG, 1997). As probabilidades ergódicas podem ser determinadas da seguinte maneira:

$$
\begin{aligned}
& \rho_{1}=\left(1-\rho_{11}\right) /\left(2-\rho_{11}-\rho_{22}\right) \\
& \rho_{2}=\left(1-\rho_{22}\right) /\left(2-\rho_{11}-\rho_{22}\right)
\end{aligned}
$$

A probabilidade de se estar no regime 1 em equilíbrio é obtida por $\rho_{1}$ e a probabilidade de se estar no regime 2 é determinada por $\rho_{2}$.

O estimador de máxima verossimilhança é utilizado para determinar os parâmetros dos MS (N)$A R(p)$. Portanto, a função de probabilidade do log do modelo com dois regimes é expressa da seguinte maneira: 


$$
\ln L=\sum_{t=1}^{T} \ln \left\{\sum_{j=1}^{2} f\left(y_{t} \mid S_{t}, y_{t-1}\right) \operatorname{Pr}\left(S_{t}=j \mid Y_{t-1}\right)\right\}
$$

Onde o termo $P_{r}\left(S_{t}=j \mid y_{t-1}\right)$ é a probabilidade de estar em cada regime. Dado $\operatorname{Pr}\left(S_{t-1}=i \mid y_{t-1}\right), \mathrm{i}=1,2$ no início do tempo $t$, as probabilidades de estar em cada regime são obtidas da seguinte maneira:

$$
\operatorname{Pr}\left(S_{t}=j \mid y_{t-1}\right)=\sum_{i=1}^{2} \operatorname{Pr}\left(S_{t}=j \mid S_{t-1}=i\right) \operatorname{Pr}\left(S_{t-1}=i \mid y_{t-1}\right) \text {, }
$$

onde $\operatorname{Pr}\left(S_{t}=j \mid S_{t-1}=i\right), \mathrm{j}=1,2 ; \mathrm{i}=1,2$ são probabilidades de transição dos elementos da matriz P, consideradas constantes. A possibilidade de se estar em regime ou em outro regime, pode ser alterada através do desempenho macroeconômico e das informações obtidas do mercado acionário.

Finalmente, a partir da matriz de transição (2) determina a duração esperada de cada regime. Quanto mais próxima a probabilidade estiver de um, leva mais tempo para mudar de outro regime. Assim a duração esperada pode ser expressa como:

$$
\operatorname{Duração~esperada~}\left(D_{i}\right)=\frac{1}{1-\rho_{i j}}
$$

O tempo de duração em cada um dos dois regimes pode ser determinado como:

$$
D_{1}=1 /\left(1-\rho_{11}\right) \quad D_{2}=1 /\left(1-\rho_{22}\right)
$$

\section{Teste de linearidade (BDS)}

Uma vez detectada que a distribuição não é normal, faz-se necessário testar o modelo para linearidade. Este teste foi desenvolvido por Brocke et al. (1996), usado para testar se as variáveis aleatórias que compõem uma série são independentes e identicamente distribuídas (IID), ou seja, pode verificar várias situações em que as variáveis não são IID, tais como não estacionariedade, não linearidade e caos determinístico. $O$ teste se baseia no conceito de correlação espacial da teoria do caos e segundo os autores a estatística BDS é formulada como:

$$
W_{m}^{n}(\varepsilon)=\frac{\sqrt{N}\left(C_{m}^{n}(\varepsilon)-\left(C_{1}^{n}(\varepsilon)\right)^{m}\right.}{\sigma_{m}(\varepsilon)}
$$

Onde $W_{m}^{n}(\varepsilon)$ converge para uma distribuição normal $\mathrm{N}(0,1)$, à medida que $\mathrm{n}$ tende para infinito. Assim sendo, os testes de hipóteses são:

$H_{0}$ : a série segue um processo iid (independente e identicamente distribuído).

$H_{1}$ : a série não segue um processo iid (independente e identicamente distribuído).

\section{METODOLOGIA}

\section{Dados}

Os dados utilizados neste estudo se referem aos índices mensais Bovespa, compreendendo o período de Janeiro de 2000 a maio de 2020, num total de 245 observações mensais. Os dados foram obtidos do site Yahoo finance. 


\section{RESULTADOS E DISCUSSÃO}

Os retornos mensais foram calculados através da fórmula: $r_{t}=\ln \left(P_{t}\right)-\ln \left(P_{t-1}\right)$. Sendo que $P_{t}$ representa o número de pontos no fechamento no dia t e $P_{t-1}$ o número de pontos no fechamento no dia anterior (t-1). As Figuras 1 e 2 mostram o comportamento das séries de cotações e retornos mensais do Ibovespa no período considerado

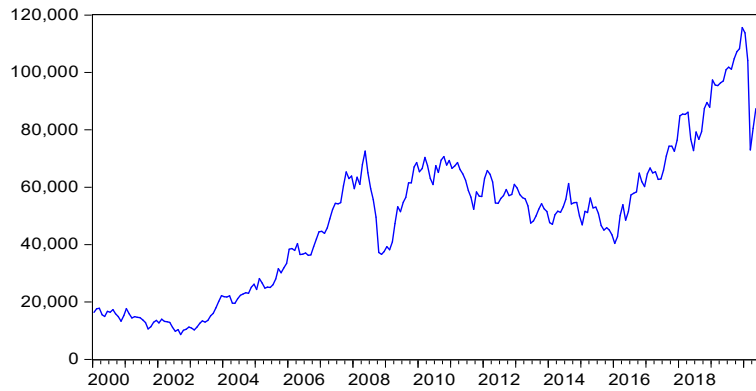

Figura 1: Cotações mensais do Ibovespa.

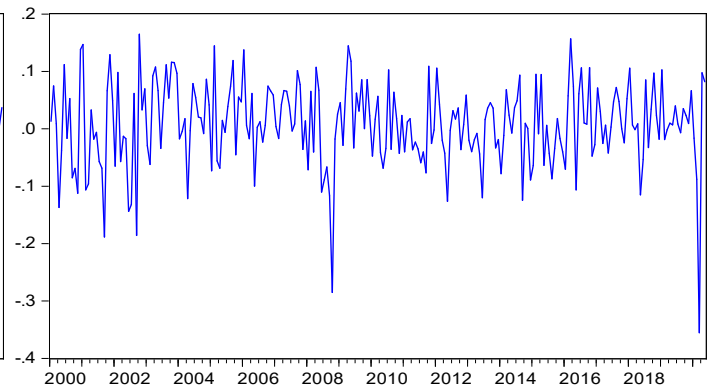

Figura 2: Retornos mensais do Ibovespa.

$\mathrm{Na}$ inspeção visual da Figura 2, dentro do período de análise, nota-se uma acentuada volatilidade nos retornos. Assim, foi preciso testar a normalidade e estacionariedade da série dos retornos do Ibovespa para aplicação do modelo MS-AR.

Algumas estatísticas descritivas básicas são apresentadas na Tabela 1. Observa-se que os retornos mensais do Ibovespa apresentam uma distribuição leptocúrtica devido ao excesso de curtose $(5,466301)$ em relação à distribuição normal $(3,0)$, ou seja, possui calda mais pesada. Verifica-se também que a série é negativamente assimétrica o que indicaria que baixas de mercado acionário são mais prováveis do que altas de mercado. Pela análise dos resultados, constata-se que tanto a média $(0,067)$ quanto a mediana $(0,0083)$ apresentaram valores próximos de zero. A variação entre o valor mínimo $(-0,355)$ e o valor de máximo $(0,165)$ mostrada pela série, pode ser explicada devido algumas oscilações significativas nos retornos dos índices. $O$ baixo valor do desvio-padrão $(0,073)$ indica que, de maneira geral, as altas variações na série ocorreram em poucas ocasiões, ou seja, em períodos de picos positivos e negativos. A estatística de Jarque et al. (1987) indicou a rejeição da normalidade da distribuição da série, com p-valor igual a zero.

Tabela 1: Sumário estatístico dos retornos do Ibovespa.

\begin{tabular}{|c|c|c|c|c|c|}
\hline Estatísticas & Média & Mediana & Máximo & Mínimo & Desvio padrão \\
\hline Valores & 0,066886 & 0,008334 & 0,164812 & $-0,355310$ & 0,073232 \\
\hline Estatísticas & Assimetria & Curtose & Jarque-Bera & $\mathrm{p}$-valor & Observações \\
\hline Valores & $-0,807975$ & 5,466301 & 8.75057 & 0,000000 & 245 \\
\hline
\end{tabular}

O Q-Q Plot representa um dos métodos gráficos mais utilizados na verificação da normalidade de séries temporais. O procedimento empregado consiste na comparação gráfica dos quantis teóricos da distribuição normal com os quantis dos dados amostrais. A Figura 3 mostra a existência de uma relação não linear entre os quantis teóricos e empíricos, bastante acentuada nas caudas das distribuições, indicando caudas mais pesadas na distribuição empírica. Assim sendo, todos os testes rejeitaram a hipótese de normalidade da série analisada. 


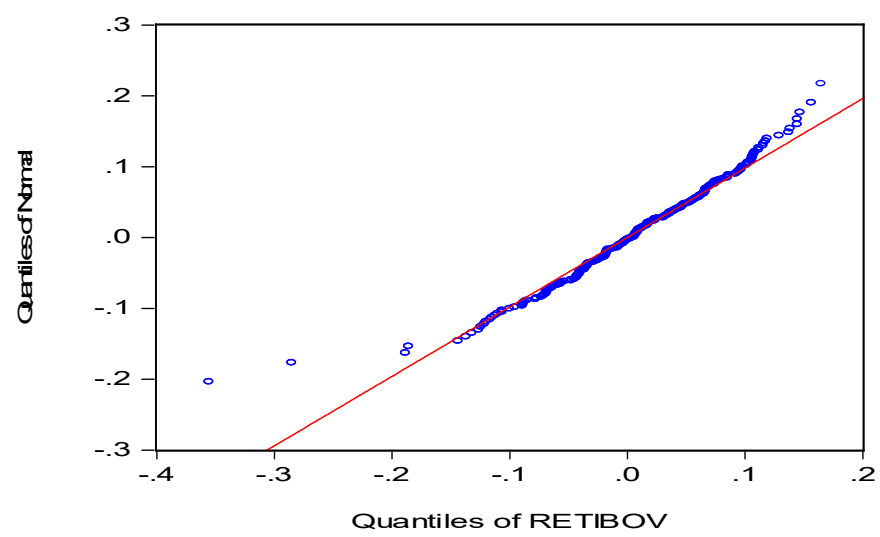

Figura 3: Plot Q-Q dos retornos do Ibovespa.

Os testes Dickey et al. (1981) Aumentado (ADF) (1979) e Phillips et al. (1988) (PP) e Kwiatkowskik, et al. (1992) (KPSS) com constante e com tendência, identificaram que as séries de retornos do lbovespa são estacionárias e não contém raízes unitárias, conforme se observa na Tabela 2.

Tabela 2: Teste de estacionaridade para a série dos retornos do Ibovespa.

\begin{tabular}{|l|l|l|l|l|l|l|}
\hline Variável & ADF & $\begin{array}{l}\text { Valor Crítico } \\
(5 \%)\end{array}$ & PP & $\begin{array}{l}\text { Valor Crítico } \\
(5 \%)\end{array}$ & KPSS & $\begin{array}{l}\text { Valor Crítico } \\
(5 \%)\end{array}$ \\
\hline Ibovespa & $-15,5307$ & $-3,4285$ & $-78,6986$ & $-3,4285$ & 00,0655 & 0,1460 \\
\hline
\end{tabular}

Antes da estimativa do modelo Markov Switching autorregressivo (MS-AR) um teste de não linearidade pode ser necessário descrever as características da série histórica dos retornos do Ibovespa. Assim, na tabela 3 mostra que os resultados apresentados indicam o efeito de não linearidade, ou seja, que as probabilidades são menores que 5\% ao nível de significância, implicando uma rejeição da hipótese nula de que a série dos retornos é linearmente dependente.

Tabela 3: Teste à independência temporal do lbovespa (BDS).

\begin{tabular}{|l|l|l|l|}
\hline Dimensão & Estatística BDS & Estatística Z & Probabilidade \\
\hline 2 & 0,01115 & 2,6263 & 0,0086 \\
\hline 3 & 0,02225 & 3,3081 & 0,0009 \\
\hline 4 & 0,01957 & 2,4509 & 0,0143 \\
\hline 5 & 0,02198 & 2,6494 & 0,0081 \\
\hline 6 & 0,02438 & 3,0572 & 0,0022 \\
\hline
\end{tabular}

O processo de modelagem e escolha dos modelos $A R(p)$, deve testar modelos diferentes e verificar a escolha do mais adequado com base nos menores valores dos critérios de informação Akaike (AIC) e Schwarz (SIC), bem como a estatística de Durbin-Watson (DW) que também detecta a presença de autocorrelação nos resíduos de uma análise de regressão, ou seja, quando DW é aproximadamente igual a 2,0, ele indica que não existe autocorrelação nos resíduos. Desta forma, após várias simulações e com os critérios mencionados anteriormente, a melhor estimativa foi para o AR (1), devido aos menores valores observados para os critérios de AIC e SIC, bem como e coeficiente de Durbin-Watson (DW) mais próximo de 2 (tabela 4).

Após a seleção do número de defasagens do componente autorregressivo (AR), foi determinar o número de regimes do modelo Markov Switching (MS), conforme apresentado na tabela 5. Assim, segundo o critério de informação Schwarz (SIC), o melhor modelo foi de dois regimes, já que apresenta o menor valor 
$(-2,3444)$.

Tabela 4: Seleção da ordem do componente autorregressivo AR(p).

\begin{tabular}{|l|l|l|l|}
\hline Modelo Autorregressivo(AR) & AIC & SIC & DW \\
\hline$A R(1)^{*}$ & 2,3826 & $-2,3540$ & 1,98 \\
\hline $\operatorname{AR}(2)$ & 2,3806 & $-2,3377$ & 1,75 \\
\hline $\operatorname{AR}(3)$ & 2,3737 & $-2,3166$ & 1,76 \\
\hline$A R(4)$ & 2,3669 & $-2,2955$ & 1,76 \\
\hline
\end{tabular}

$\left({ }^{*}\right)$ melhor modelo ajustado

Tabela5: Determinação do número de regimes através do critério (SIC)

\begin{tabular}{|l|l|l|}
\hline Modelo & Regimes & SIC \\
\hline MS & 4 & $-2,2216$ \\
\hline MS & 3 & $-2,2819$ \\
\hline MS & 2 & $-2,3444$ \\
\hline
\end{tabular}

$\left({ }^{*}\right)$ melhor número de regime do modelo MS-AR.

Na tabela 6 observa-se as estimativas do modelo pelo método da máxima verossimilhnaça, através do software OxMetrics 6.0. O melhor modelo ajustado refere-se ao MS(2)-AR(1), a média e a variância se modificam de acordo com o regime de estado. O regime (1) exprime uma média dos retornos positiva do Ibovespa em conjunto com uma baixa volatilidade. Já no regime (2) mostra um resultado da média negativa e uma alta volatilidade nos retornos do Ibovespa. No regime 1 o retorno médio mensal estimado é de 1,49\% $(19,42 \%$ ao ano) com uma variância de 0,028 . Já o regime 2 identifica retorno médio mensal negativo de 2,52\% (-34,8\% ao ano) com uma variância de 0,108.

No modelo de mudança de regime markoviano, foi possível identificar um regime com retornos negativos e com variância elevada (alta volatilidade ou mercado em baixa) e outro regime com retornos positivos menor variância (baixa volatilidade ou mercado em alta).

Portmanteau indicam que não há presença de autocorrelação dos resíduos. Os resultados dos testes ARCH-LM sugerem a aceitação da hipótese de homocedasticidade do modelo. Quanto aos testes de normalidade Jarque et al. (1987) não se rejeita a hipótese de normalidade. Assim, o modelo apresenta um diagnóstico positivo e um adequado ajuste demonstrado nos resultados dos diversos testes realizados no presente estudo.

Na matriz de transação e persistência dos regimes, verifica-se que o regime atual 1 é mais persistente, ou seja, a probabilidade de permanecer neste regime em período posterior é de aproximadamente de $98,4 \%$, e a de mudar para o regime 2 é da ordem de 6,51\%. No regime 2 a probabilidade de continuar neste regime no período $t+1$ é de 93,49\%, enquanto a probabilidade de trocar para o regime 1 é de 1,61\%. Assim, para o período de janeiro de 2000 a maio de 2020, a duração esperada do regime atual 1 é de 99 meses. Já no regime 2 a duração estimada é de 15 meses. A probabilidade ergódicas nos períodos de baixa volatilidade é de $81,56 \%$ e $18,44 \%$ nos períodos de alta volatilidade.

A partir das probabilidades estimadas pode-se obter as datas específicas dos regimes de baixa volatilidade (1) e de alta volatilidade (2), os quais apresentados na tabela 7. 
Tabela 6: Estimativa do modelo MS(2)-AR(1) para periocidade mensal (Janeiro 2000 a Maio 2020)

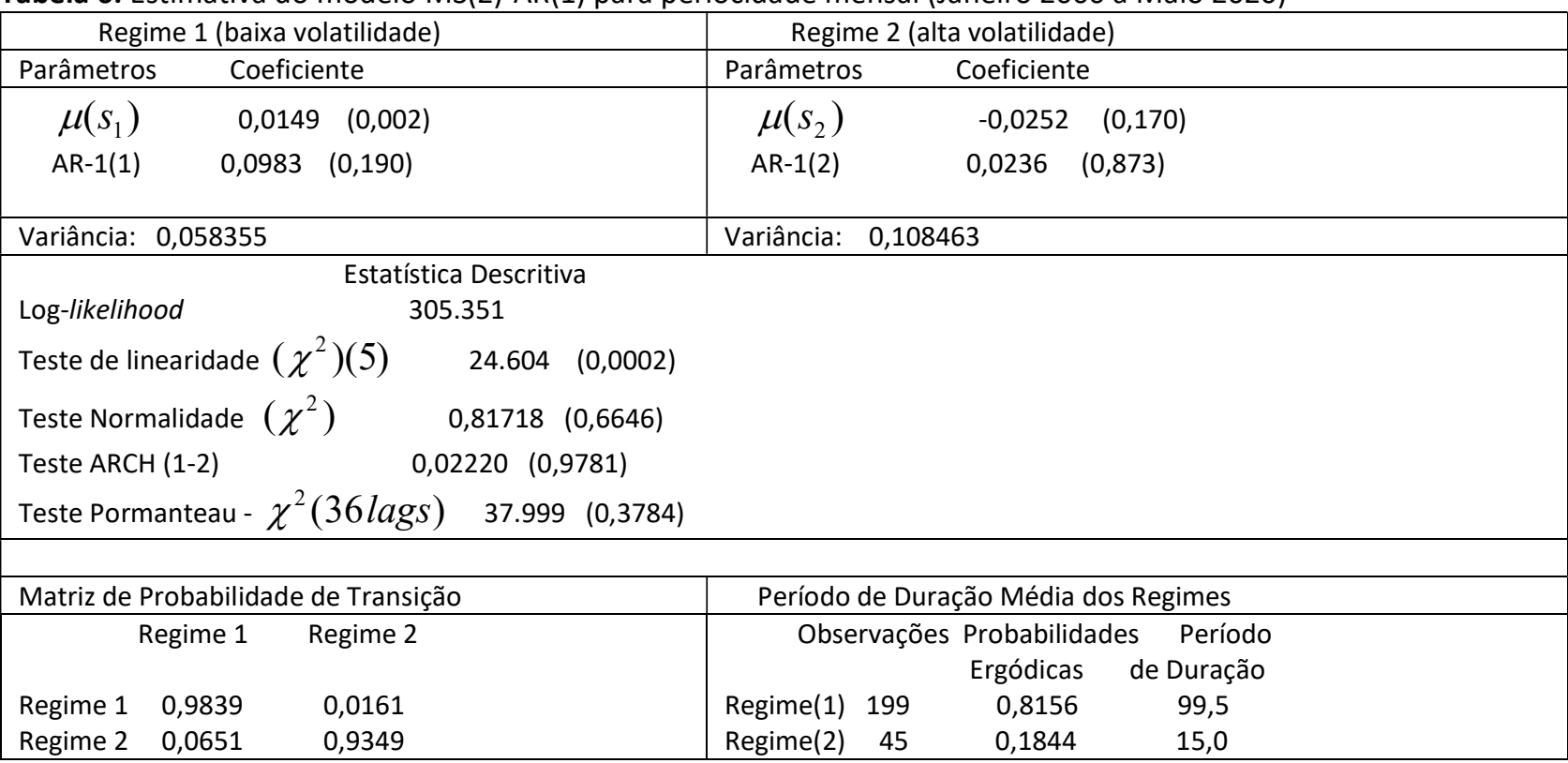

Tabela 7: Datas específicas dos regimes: Modelo MS(2)-AR(1)

\begin{tabular}{|c|c|c|c|c|c|}
\hline \multicolumn{3}{|c|}{ Regime 1 (baixa volatilidade) } & \multicolumn{3}{|c|}{ Regime 2 (alta volatilidade ) } \\
\hline Período & Meses & Probalidade & Período & Meses & Probabilidade \\
\hline $2002(12)-2008(4)$ & 65 & 0,945 & $2000(2)-2002(11)$ & 34 & 0,931 \\
\hline $2008(12)-2020(1)$ & 134 & 0,969 & $\begin{array}{l}2008(5)-2008(11) \\
2020(2)-2020(5)\end{array}$ & $\begin{array}{l}7 \\
4\end{array}$ & $\begin{array}{l}0,835 \\
0,861\end{array}$ \\
\hline
\end{tabular}

Entre o início de 2000 e final de 2003, verifica-se uma tendência de queda devido ao cenário interno e externo mais instável, o atentado terrorista de 11/09/2001 e o momento de transição da política brasileira (vitória de Lula na eleição presidencial 2002).

Em setembro de 2008 teve início a uma queda significativa no índice Bovespa, ocasionada pela crise do subprime deflagrado com a falência de um dos bancos de investimentos dos EUA, o Lehman Brothers, desencadeando uma crise nas bolsas de valores intenacionais.

No início de janeiro de 2020, o lbovespa teve um impacto negativo devido ao coronavírus, que vem gerando fortes turbulências nos mercados mundiais e as políticas de isolamento para conter o avanço da pandemia (covid-19), refletindo na economia os efeitos da paralisação de diversas atividades econômicas.

A figura 4 mostra o comportamento dos retornos, das probabilidades suavizadas e previstas para os regimes de estado do Ibovespa. A probabilidade suavizada considera as informações da amostra completa.

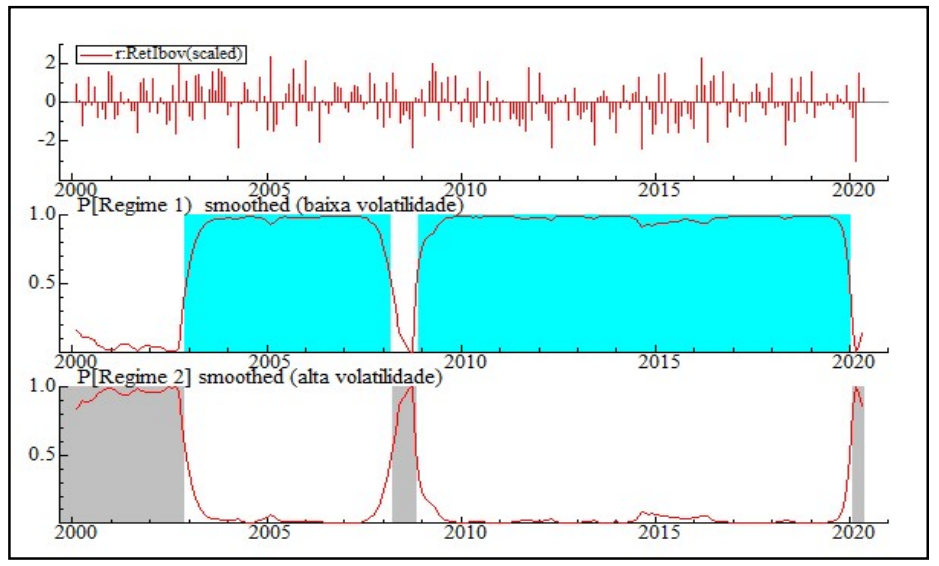

Figura 4: Série dos retornos do Ibovespa e as Probabilidades suavizadas do modelo MS(2) - AR(1). 


\section{CONCLUSÕES}

O objetivo do estudo foi analisar as alterações nos retornos do lbovespa, entre janeiro de 2000 e maio de 2020, utilizando o modelo Autorregressivo com Mudanças Markovianas desenvolvido por Hamilton (1989). O melhor modelo ajustado refere-se ao MS(2)-AR(1), a média e a variância se modificam de acordo com o regime de estado. O regime (1) exprime uma média dos retornos positiva do Ibovespa em conjunto com uma baixa volatilidade. Já no regime (2) mostra um resultado da média negativa e uma alta volatilidade nos retornos do Ibovespa. No regime 1 o retorno médio mensal estimado é de 1,49\% (19,42\% ao ano) com uma variância de 0,028 . Já o regime 2 identifica retorno médio mensal negativo de $-2,52 \%(-34,8 \%$ ao ano) com uma variância de 0,108.

No modelo de mudança de regime markoviano, foi possível identificar um regime com retornos negativos e com variância elevada (alta volatilidade ou mercado em baixa) e outro regime com retornos positivos menor variância (baixa volatilidade ou mercado em alta).

\section{REFERÊNCIAS}

ANG, A.; BEKAERT, G.. International Asset Allocation with Regime Shifts. Review of Financial Studies, v.15, p.11371187, 2002.

ASSOE, K. G.. Regime Switching in emerging stock market returns. Multinational Finance Journal, v.2, n.2, p.101-132, 1998.

BROCK, W. A.; DECHERT, W. D.; SCHEINKMAN, J.. A Test for Independence Based on the Correlation Dimension. Econometric Reviews, v.15, n.3, p.197-235, 1996. DOI: https://doi.org/10.1080/07474939608800353

DICKEY, D. A.; FULLER, W. A.. Likelihood ratio statistics for autoregressive Time series with unit root. Econometrica, v.49, n.4, p.1057-1072, 1981.

HAMILTON, J.. A New Approach to the Economic Analysis of Nonstationary Time Series and the Business Cycle.

Econometrica, v.57, p.357-384, 1989.

ISMAIL, M. T.; ISA, Z.. Modelling exchange rates using regime switching models. Sains Malaysiana, v.35, n.2, p. 55-62, 2006.

JARQUE, C.; BERA, A.. Test for normality of observations and regression residuals. International Statistical Review, v.55, n.2, p.163-172, 1987.

KIM, C.-J.. Dynamic linear models with Markov-switching.. Journal of Econometrics, v.60, p.1-22, 1994.

KROLZIG, H. M.. Markov-Switching vector autoregressions: modelling, statistical inference, and application to business cycle analysis. Berlin: Springer, 1997.

KROLZIG, H. M.; TORO, J.. Multiperiod forecasting in stock markets: a paradox solved. Decision Support Systems, v.37, n.4, p.531-542, 2004.

KWIATKOWSKIK, D.; PHILLIPS, P. C. B.; SCHMIDT, P.; SHIN, Y.. Testing the null hypothesis of stationarity against the alternative of a unit root: How sure are we that economic time series have a unit root?. Journal of Econometrics, v.54, n.1, p.159-178, 1992.

MAHJOUB, M. R.; CHASKMI, S. A. N.. Identification the Periods of Formation and Bursting of Speculative Bubbles in Iranian Stock Market Using Quantitative Models. Advances in mathematical finance \& applications, v.4, n.4, p.129-140, 2019.

NORDEN, S. V.; SCHALLER, H.. Regime Switching in Stock Market Returns. Berlin: Econometrics, 1995.

OLIVEIRA, A. B.; PEREIRA, P. L. V.. Asset allocation with markovian regime switching: Efficient frontier and tangent portfolio with regime switching. Brazilian Review of Econometrics, v.38, n.1, p.97-127, 2018.

PHILLIPS, P. C. B.; PERRON, P.. Testing for a unit root in time series regression. Biometrika, v.75, n.3, p.335-346, 1988.

SAFAEI, M.; MOSTAFAEI, H.. Point Forecast Markov Switching Model for U.S. Dollar/Euro Exchange Rate. Sains Malaysiana, v.41, n.4, p.481-488, 2012.

SCHWERT, W.. Business Cycles, Financial Crises, and Stock Volatility. Carnegie Rochester Conference Series on Public Policy, v.31, p.83-126, 1989.

WINK JÚNIOR, M. V.; ZUANAZZI, P. T.. A sensibilidade de ativos em diferentes ambientes de risco: uma análise para empresas gaúchas. Ensaios FEE, v.35, n.1, p.231-248, 2014.

A CBPC - Companhia Brasileira de Produção Científica (CNPJ: 11.221.422/0001-03) detém os direitos materiais desta publicação. Os direitos referem-se à publicação do trabalho em qualquer parte do mundo, incluindo os direitos às renovações, expansões e disseminações da contribuição, bem como outros direitos subsidiários. Todos os trabalhos publicados eletronicamente poderão posteriormente ser publicados em coletâneas impressas sob coordenação da Sustenere Publishing, da Companhia Brasileira de Produção Científica e seus parceiros autorizados. Os (as) autores (as) preservam os direitos autorais, mas não têm permissão para a publicação da contribuição em outro meio, impresso ou digital, em português ou em tradução. 